\title{
Characterisation of an electrical heating method for metallic-coated optical fibres for distributed sensing applications
}

\author{
Xin Lu*, Marcelo A. Soto, Luc Thévenaz \\ EPFL Swiss Federal Institute of Technology, Institute of Electrical Engineering, \\ SCI STI LT, Station 11, CH-1015 Lausanne, Switzerland \\ *E-mail: xin.lu@epfl.ch
}

\begin{abstract}
In several applications a temperature contrast between the sensing fibre and the environment is required to detect changes in the environmental heat capacity. For this purpose the process of electrical heating in metallic-coated fibres is theoretically analysed and modelled in steady-state conditions based on the thermal energy generated by resistive heating and the losses induced by convection and radiation. The impact of ambient temperature and pressure is investigated. The proposed model for the thermal exchange is experimentally validated using a high-resolution Brillouin distributed fibre sensor, which is used to measure the longitudinal profile of the temperature reached by electrical heating along an Alcoated optical fibre.
\end{abstract}

Keywords: Fibre optics, optical fibre sensors, distributed fibre sensing, electrical heating

\section{INTRODUCTION}

Nowadays optical fibre sensing is successfully commercialised and applied to different fields due to the advantages of optical fibres, such as small size and low weight, as well as due to distributed sensing capabilities. As the application area of fibre sensors expands, the use of traditional passive fibres could fail to meet some special requirements needed under specific operation conditions, and where the use of active heating methods might be beneficial for improving the sensing response. The thermal exchange between the heated fibre and the surrounding environment could actually bring meaningful information, enabling fibre sensors to measure new physical quantities. For instance, self-heated fibres have been applied to measure Hydrogen concentration ${ }^{1}$, detect air flow as an anemometer ${ }^{2}$ and monitor the moisture of soil ${ }^{3}$. Furthermore, active heating could increase the working temperature of the fibre, so that the low sensitivity shown by some fibre sensors at low-temperature (e.g. cryogenic) conditions can be improved ${ }^{4}$.

Active fibre heating methods can be classified into optical and electrical heating. For optical heating, a high-power optical beam is launched into the sensing fibre at a specifically chosen wavelength and a small portion of the light is absorbed and converted into thermal energy to heat the fibre ${ }^{1}$. Although optical heating keeps the fibre immunity to electromagnetic interferences (EMI) and environmental conductivities, the heating efficiency turns out to be very low and uneven ${ }^{2}$. This is because optical fibres are essentially transparent, and if an optical source with a particular wavelength is used to maximise the power absorption, a significant longitudinal temperature gradient would be induced due to the exponential decay of the optical power propagating in the fibre. This leads to a non-uniform temperature profile along the fibre ${ }^{2}$. On the other hand, for electrical heating, an electric current is circulated along metallic coating or tube surrounding the fibre, so that the generated heat by the pure resistive Joule effect raises the fibre temperature ${ }^{2,5}$. Compared to optical heating, electrical heating methods demonstrate high energy efficiency and a more uniform temperature profile. However, this method is more vulnerable to EMI and environmental perturbations ${ }^{2}$. So far, both methods have been used in point discrete sensors and in short-range distributed fibre sensors; however electrical heating is the only candidate for long-range distributed sensing due to its high efficiency and longitudinal uniformity.

In this paper, a thermal model is built for the electrical fibre heating process and its behaviour is analysed under different temperature and pressure conditions. Simulated result show the heating efficiency increases abruptly below a certain threshold pressure, where the dominant heat loss factor changes from natural convection to radiation. The model is validated by measuring the thermal response of an Al-coated optical fibre using a high-resolution Brillouin optical time domain analyser (BOTDA). An excellent matching between experimental and simulation results is verified.

Sixth European Workshop on Optical Fibre Sensors, edited by Elfed Lewis, Proc. of SPIE Vol. 9916, 991620 - (c) 2016 SPIE · CCC code: 0277-786X/16/\$18 · doi: 10.1117/12.2236951 


\section{THEORY}

When an electric current passes through a conductor, the interaction between the charged particles and the atomic ions in the conductor releases heat. According to the Joule's first law, the generated thermal energy is proportional to the resistive impedance of the conductor and the square of the circulating current ${ }^{5}$. To realise electrical heating an electrical current is directly conducted in the coating of a metallic-coated fibre. The electrical energy is converted into thermal energy in the coating, so that the fibre turns out to be heated by simple thermal conduction.

The thermal transfer in this electrical heating method is schematically shown in Fig. 1: part of the heat generated in the metal coating transfers to the optical fibre by thermal conduction and the rest is lost into the air by convection and radiation. The whole process can be modelled as a silica cylinder with a thin-film thermal source on its surface and surrounded by air ${ }^{6}$. The fibre radius is assumed very small compared to the fibre length used in long-range distributed sensing. As a result, the heat flow in the radial direction can be neglected. Thus the energy exchange for this metal coated fibre is described by ${ }^{6}$ :

$$
\frac{\partial T(x, t)}{\partial t}=\kappa \frac{\partial T^{2}(x, t)}{\partial x^{2}}+\frac{P_{i n}(x, t)-P_{o u t}(x, t)}{d c_{p} \pi(r+b)^{2}},
$$

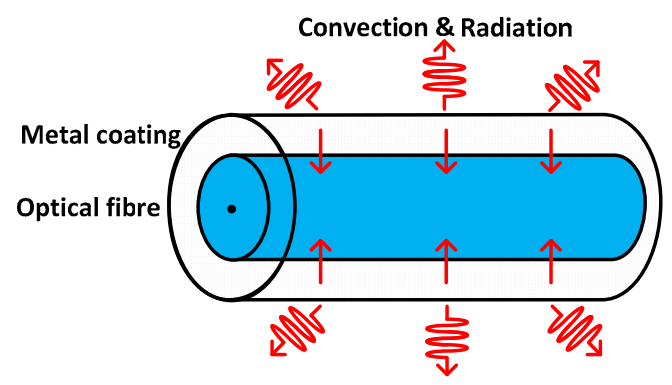

Figure 1. Thermal transfer for a metallic coated fibre with electrical heating.

where $T$ is the fibre temperature, $x$ is the distance along the fibre, $t$ represents time, $r$ is the radius of the uncoated fibre, $b$ is the thickness of the coating and $\kappa, d$ and $c_{\mathrm{p}}$ are the thermal diffusivity, density and constant pressure heat capacity of silica, respectively. $P_{\text {in }}$ is the heating power density from the coating, which is governed by Joule's first law, while $P_{\text {out }}$ is the thermal power loss density by convection and radiation.

Considering that distributed optical fibre techniques are usually applied for pseudo-static measurements, only the steady state solution of the thermal exchange is considered here. Under this condition the left-hand side term of Eq. (1) becomes zero. In addition, for the sake of simplicity, the metal coating and optical fibre are assumed to be longitudinally uniform, so that the increased temperature is identical at any fibre location. As a result, there is no thermal conduction along the fibre, leading to $\partial T^{2} / \partial x^{2}=0$. Eventually, the input energy is totally dissipated into the surrounding environment as Eq. (1) becomes $P_{\text {in }}-P_{\text {out }}=0$. The analysis above shows that the energy conservation is reached at the steady-state condition, which is described by the following equation per unit length ${ }^{5}$, equating electrical heating power with thermal dissipation:

$$
I^{2} \frac{\rho}{\pi b(2 r+b)}=2 \pi(r+b)\left[\delta \varepsilon\left(T^{4}-T_{0}^{4}\right)+H\left(T-T_{0}\right)\right]
$$

where $I$ is the applied current, $\rho$ is the resistivity of the metal coating, $H$ is the convective coefficient, $T_{0}$ is the ambient temperature, $\delta$ is the Stefan-Boltzmann constant and $\varepsilon$ is the surface emissivity.

At ambient pressure, the heat loss is dominated by natural convection. The convection strength is determined by the convective coefficient $H$, which according to the literature is typically above $100 \mathrm{~W} /\left(\mathrm{m}^{2} \mathrm{~K}\right)$ for different fibre coatings ${ }^{6,7}$. A dimensionless quantity called Biot number, $B i=H \cdot(r+b) / 2 k$ (with thermal conductivity $k$ of silica) indicates the relative importance of heat transfers inside and at the surface of the fibre. It is calculated to be 0.0031 , which is much smaller than 1 , meaning that the radial thermal transfer within the metallic-coated fibre can be neglected ${ }^{6}$. On the other hand, when the external pressure is reduced such that another index called Knudsen number turns larger than 1, convection and heat conduction becomes negligible compared to radiation, leading to a heat transfer coefficient typically falling below $0.01 \mathrm{~W} /\left(\mathrm{m}^{2} \mathrm{~K}\right)$ for very low pressures ${ }^{8}$. Consequently, the heating is as expected more efficient in vacuum. In addition, when the natural convection dominates the process, Eq. (2) linearly depends on the temperature difference, meaning that the heating efficiency is independent of the ambient temperature. However, when radiation plays the key role in the thermal loss, the relation turns strongly dependent on $T_{0}$, making the heating more efficient at low temperatures.

\section{SIMULATION AND EXPERIMENT}

The electrical heating of a standard single-mode fibre with Al-coating is simulated based on the proposed thermal model in Eq. (2). The fibre radius $r$ is $62.5 \mu \mathrm{m}$, and the thickness of the coating $b$ is $25 \mu \mathrm{m}$. Since aluminium gets oxidized 
easily in open air, the emissivity of the aluminium oxide 0.15 is used in the simulation ${ }^{9}$. The $\mathrm{Al}$ resistivity is $2.733 \times 10^{-8}$ $\Omega \mathrm{m}$ at $300 \mathrm{~K}$ and $1.62 \times 10^{-9} \Omega \mathrm{m}$ at $70 \mathrm{~K}^{10}$ and is assumed constant during the heating for simplicity.

Figure 2 reports the simulation results for the $\mathrm{Al}$ coated fibre, showing the temperature raise as a function of the currents under different working conditions. Figure 2(a) shows that the fibre is barely heated at normal ambient pressure (high convective coefficient) because most of the generated thermal energy is quickly dissipated through natural convection. It is observed that electrical heating is much less efficient under cryogenic conditions due to the decreased $\mathrm{Al}$ resistivity. In high vacuum (less than $10^{-2} \mathrm{~Pa}$ ) when thermal dissipation is dominated by radiative losses and the convection turns negligible (low $H$ ), the heating becomes much more efficient, as shown in Fig. 2(b). In this case, a higher temperature increase is observed under cryogenic conditions for the same current despite of the low metal resistivity, demonstrating the key role played by ambient temperature when radiative dissipation dominates.
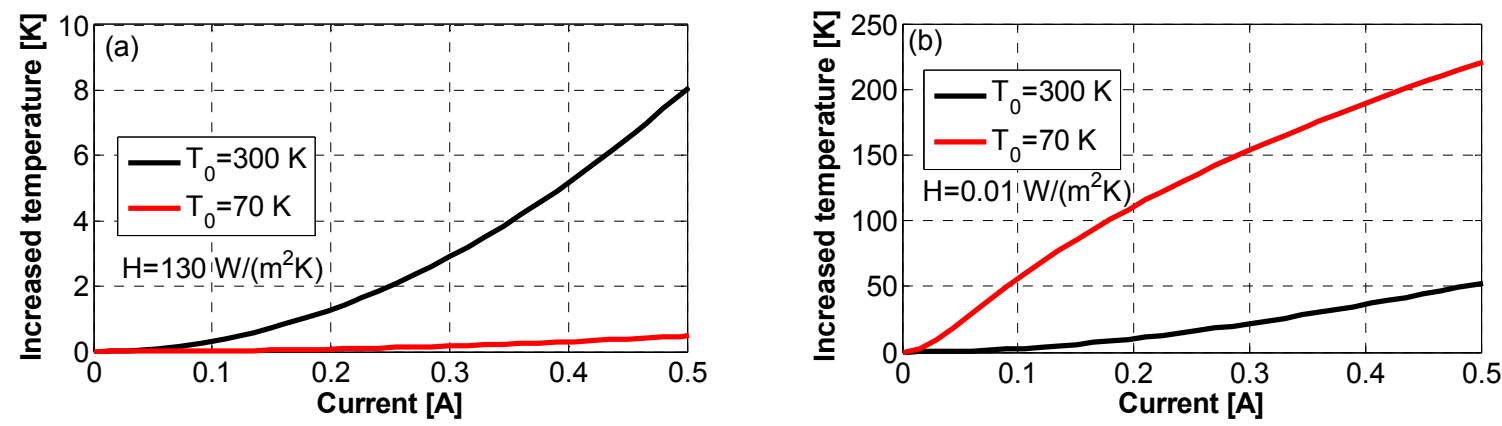

Figure 2. Fibre temperature raised by electrical heating (simulation results) as a function of the currents, for different ambient temperatures, when using Al coating, at (a) 1 atmosphere pressure and (b) vacuum. Temperature scales are different in the 2 cases

In order to validate the model and theoretical results, the temperature change induced by electrical heating has been noninvasively measured in an Al-coated optical fibre by using a Brillouin distributed fibre sensor. Due to the short fibre length $(1.3 \mathrm{~m})$, a double-pulse scheme is implemented in a standard BOTDA to achieve high spatial resolution ${ }^{11}$. Optical pulses of $2 \mathrm{~ns}$ are used, corresponding to $20 \mathrm{~cm}$ spatial resolution. The Brillouin temperature sensitivity of this fibre is characterised to be $1.49 \mathrm{MHz} / \mathrm{K}$. It is $\sim 50 \%$ higher than the sensitivity of the standard single mode fibre because the thermal expansion of the $\mathrm{Al}$ coating introduces additional strain that contributes the Brillouin frequency shift.
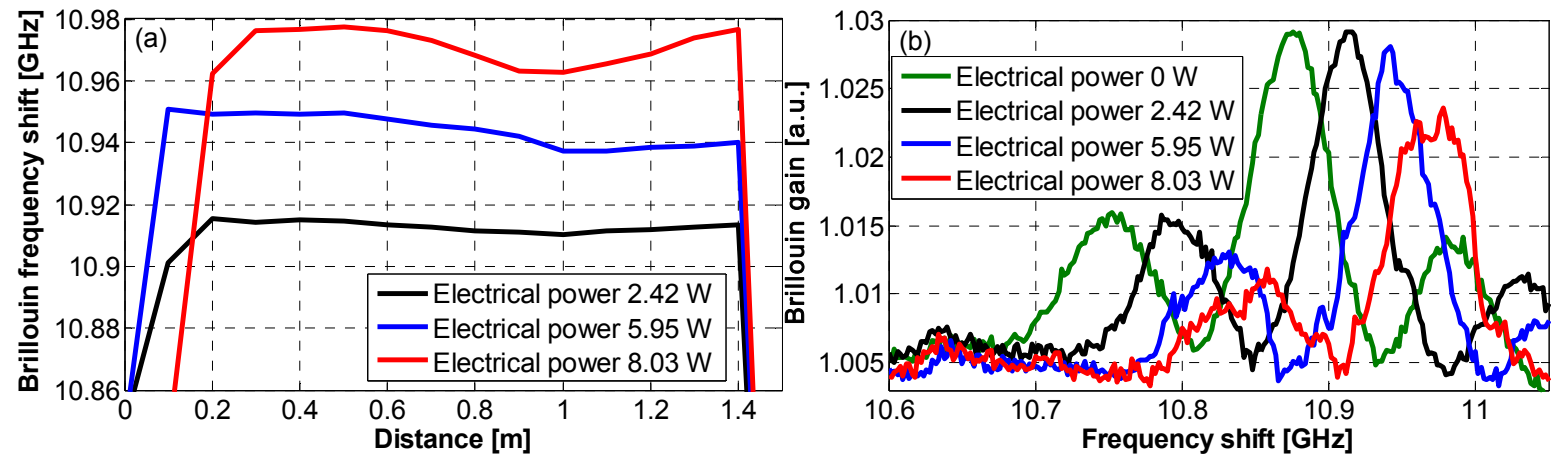

Figure 3. a. Brillouin frequency and b. averaged Brillouin spectrum along the Al-coated fibre with different electrical power.

In practice, the metal resistance is dependent on temperature and it becomes more complicated to solve Eq. (2). To avoid this problem, the input power is expressed as the product of current $I$ and voltage $U$ instead of $I^{2} \rho /[\pi b(2 r+b)]$ as shown in Eq. (2). Hence the Brillouin frequency and gain spectrum of the coated fibre are measured under different electrical powers, as shown in Fig. 3, regardless of any metal resistivity change. It is obvious that the Brillouin frequency is upshifted as a result of the temperature rise induced by the electrical power. It is interesting to notice in Fig. 3(a) that the Brillouin frequency shows longitudinal fluctuations along the fibre, especially when using high electrical powers. This could be explained by the longitudinal non-uniformity of the coating. This also indicates that using distributed temperature sensing and electrical heating in metallic-coated fibres could lead to a reliable way to measure the uniformity of the coating. Figure 3(b) shows the averaged Brillouin gain spectrum, which presents some distortions at high electrical power as a result of the non-uniform temperature along the entire fibre sample. Note that the spectrum 
also shows sidelobes as a result of the interference among the acoustic waves generated by the successive optical pulses, as expected from a double-pulse BOTDA system ${ }^{11}$.

To compare the simulated results with the experimental ones, and thus to validate the proposed model, the convective coefficient needs to be determined in Eq. (2). Usually, $H$ is obtained from the transient time required to heat the fibre up to a steady-state condition ${ }^{5-7}$ (typically in the order of a few seconds). Since standard Brillouin fibre sensors are unable to measure such a quick transient time, we have here used a typical value reported in the literature, indicating a constant coefficient with a linear correction with temperature ${ }^{7}: H=130+0.299 \cdot\left(T-T_{0}\right)$. Using the above-measured temperature sensitivity of $1.49 \mathrm{MHz} / \mathrm{K}$, the temperature changes obtained by simulation and experiment are plotted in Fig. 4 as function of electrical power. A good agreement is observed between the simulation and experiment, thus validating the model. It is however possible to see a small discrepancy when using an electrical power above $6 \mathrm{~W}$, which may be accounted to the nonuniformity of the coating.

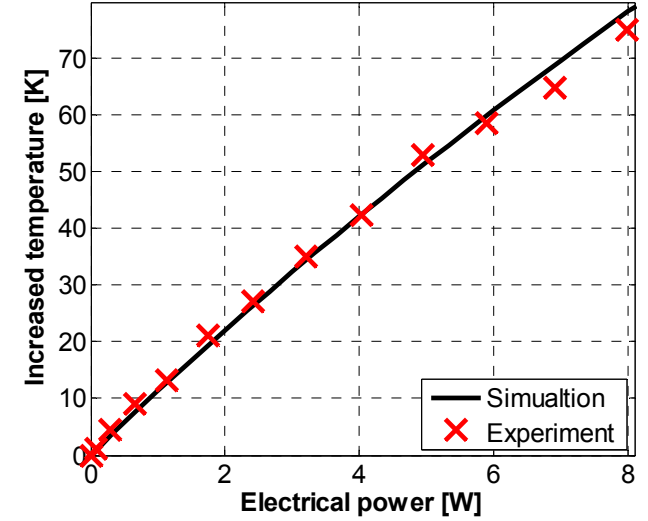

Figure 4. Comparison of the increased temperature obtained by simulation and experiment.

\section{CONCLUSION}

A thermal model is built to describe the energy exchange of metallic-coated fibres for electrical heating. The influences of environmental temperature and pressure on the heating performance have been investigated. High efficiency is found in vacuum because natural convection vanishes at low pressure and radiation dominates. The proposed model has been experimentally validated using a high-resolution Brillouin distributed temperature sensor, incidentally demonstrating the potential of distributed sensing to measure the longitudinal non-uniformity and the thickness of the coating.

This work was performed within the framework of ITER-EPFL Task Agreement (Project IO/CFT/11/5840/RTS). The views and opinions expressed herein do not necessarily reflect those of the ITER organization. The authors would like also to thank AMS Technologies and Fiberguide for providing the Al-coated fibre sample used in this experiment.

\section{REFERENCES}

[1] Buric, M., Chen, T., Maklad, M., Swinehart, P.R., and Chen, K.P., "Multiplexable low-temperature fiber Bragg grating hydrogen sensors," IEEE Photon. Technol. Lett. 21(21), 1594-1596 (2009).

[2] Chen, T., et al., "Distributed flow sensing using optical hot-wire grid," Opt. Express, 20(8), 8240-8249 (2012).

[3] Zornoza, A. M., Loheide, I., Steven, P., "Heated distributed temperature sensing for field scale soil moisture monitoring," Groundwater, 50(3), 340-347 (2012).

[4] Lu, X., Soto, M. A., and Thévenaz, L., "MilliKelvin resolution in cryogenic temperature distributed fibre sensing based on coherent Rayleigh scattering," Proc. SPIE 9157, 91573R, (2014).

[5] Li, L., Geng, J., Yhao. L., Chen, G., Chen, G., Fang, Z., and Lam, F. C., "Response characteristics of thin-filmheated tunable fiber Bragg gratings," IEEE Photon. Technol. Lett. 15(4), 545-547 (2003).

[6] Rogers, A. J., Kuo, P., Ahuja, A., Eggleton, B., Jackman, R., "Characteristics of heat flow in optical fiber devices that use integrated thin-film heaters," Appl. Opt. 39(28), 5109-5116 (2000).

[7] Salamon, T. R., Rogers, J. A., Eggleton, B. J., "Analysis of heat flow in optical fiber devices that use microfabricated thin film heaters," Sensor Actuat. A-Phys. 95(1), 8-16 (2001).

[8] Tanaka, Y., Furusawa, T., Nakauchi, M., Nagashima, K., "Heat transfer characteristics under cryogenic, low pressure environments," Physica C Supercond. 469(15), 1862-1865 (2009).

[9] Suryawanshi, C.N., Kim, T. and Lin, C.T., "An instrument for evaluation of performance of heat dissipative coatings," Rev. Sci. Instrum., 81(3), 035105 (2010).

[10] Desai, P. D., James, H. M., Ho, C. Y., "Electrical resistivity of aluminum and manganese," J. Phys. Chem. Ref. Data, 13(4), 1131-1172 (1984).

[11] Soto, M. A., Chin, S., Thévenaz, L., "Double-pulse Brillouin distributed optical fiber sensors: analytical model and experimental validation," Proc. SPIE 8421, 842124 (2012). 